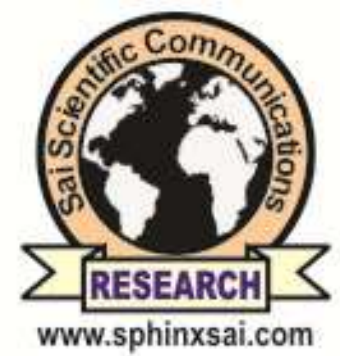

International Journal of PharmTech Research CODEN (USA): IJPRIF, ISSN: 0974-4304, ISSN(Online): 2455-9563

\title{
Right Ventricular Outflow Tract Fractional Shortening (RVOT FS) As Echocardiography Parameter for Assessment Right Ventricular Function in Systolic Heart Failure
}

\author{
Safni Marlina* ${ }^{* 1}$ Andre P. Ketaren, Cut A. Andra, \\ Harris Hasan, Ali N. Nasution, Yuke Sarastri
Departemen Kardiologidan Kedokteran Vaskular, Universitas Sumatera Utara, Rumah Sakit Umum Pusat Haji Adam Malik, Medan, Indonesia

\begin{abstract}
Background: Right ventricular function is an important evaluation in echocardiography assessment. Right ventricular function has important prognostic and therapeutic implications especially in heart failure. Right ventricular performance defines the prognosis in patients with heart failure but the assessment still has challenge because of its complex geometry and the interrelationship with the left ventricle. Right Ventricle Outflow Tract Fractional Shortening (RVOT FS) is a new parameter echocardiography, simple and easy to evaluate right ventricular function in patients with heart failure. Methods: This is a cross sectional study of 84 patients heart failure who has ejection fraction $<50 \%$ and undergo routine echocardiography in Haji Adam Malik Hospital since August 2018 until October 2018. RVOT FS was measured by M-mode from parasternal short-axis view at aortic valve level with magnified images of RVOT, and the cursor was aligned perpendicular to the anterior RVOT wall. Then, we measured RVOT end-diastolic (RVOT ED) and RVOT end-systolic (RVOT ES) diameters. Results: The cut-off value of RVOT FS $\leq 25.5$ was $88.4 \%$ sensitive and $82.9 \%$ specific to identify patients with impaired RV function in patients with heart failure. There was positive correlation between RVOT FS, TAPSE and S' with RV FAC. TAPSE has strongest correlation with RV FAC ( $\mathrm{r}=0.788, \mathrm{P}<0.001)$, $\mathrm{S}$ ' also showed positive correlation with $\mathrm{RV}$ FAC $(r=0.703, P<0.001)$ and RVOT FS $(r=0.644, \mathrm{P}<0.001)$. Conclusion : The results of this study show that the values of RVOT FS can be used as a new parameter echocardiography for assessment right ventricular function in patients with systolic heart failure.

Keyword : Right Ventricular Outflow Tract (RVOT), Right Ventricle, Systolic Heart Failure.
\end{abstract}

\section{Introduction}

In the past decades, right ventricular (RV) function was still neglected and less got attention than left ventricle. ${ }^{1,6}$ The right ventricle was considered forgotten chamber and rarely involved with epidemic heart disease such as ischemic heart disease and cardiomyopathy. In 1616, the role of right ventricular function was

Safni Marlina et al /International Journal of PharmTech Research, 2019,12(1): 57-62.

DOI: http://dx.doi.org/10.20902/IJPTR.2019.120108 
first described by Sir William Harvey through his writing "The MotuCordis". The role of RV functionwas increasingly described in heart failure, right heart myocardial infarction, congenital heart disease and pulmonary hypertension. Assessment of right ventricular function is the most important evaluation of echocardiography to understand pathophysiology of heart failure. In recent years, right ventricular function has been found to have important prognostic and therapeutic implications especially in heart failure. ${ }^{2,3,6}$

Echocardiography, non invasive, widely available, inexpensive, and has no side effect, is the modality of choice for assessment morphology and function of right ventricle in clinical practice. ${ }^{4,5}$ Echocardiography of right ventricular function in heart failure has been challenge because of its geometry complex and interrelationship with the left ventricle. In the recent studies have shown the significance of right ventricular (RV) function on the outcome in patients with left ventricular dysfunction (LVSD). ${ }^{1}$ Therefore, an additional measurement to evaluate right ventricular functionwhose simple, easy and applicable is greatly needed.

Right Ventricular Outflow Tract (RVOT) has superficial circumferential muscle fibers which causes radial contraction in systolic phase. ${ }^{2}$ The anatomy of RV has three main parts such as inflow, apical trabeculated and infundibulum (outflow tract). The RVOT contributes $20 \%$ of right ventricular contraction. Surgeons always estimate right ventricular function in cardiac surgery through contraction of RV. In the recent studies, RVOT Fractional Shortening (RVOT FS) is the echocardiography parameter whose easy and applicable for measuring right ventricular function in heart failure. ${ }^{2,3,8}$ The aim of this study to evaluate new echocardiography parameter RVOT FS for assessment right ventricular function in heart failure.

\section{Methods}

This is a cross sectional study to correlate RVOT FS as simple parameter to evaluate RV function with Right Ventricle Fractional Area Change (RV FAC).RV FAC < 35\% shows impaired RV function and RV FAC $>$ shows normal RV function. The study population consist of 84 patients heart failure who underwent routine echocardiography in Adam Malik Hospital since July 2018 until October 2018. The inclusion criteria was heart failure patients who has ejection fraction $<50 \%$ and aged 18-70 years old. The patients had structural valve heart disease, congenital heart disease, pulmonary arterial hypertension and poor echo window was excluded.

The patient's data were filled in sheets containing clinical data and standard echocardiography result with RVOT FS value from transthoracic echocardiography which has been performed by cardiology residents. Echocardiography examinations were performed using Medison Accuvix V 10 and Vivid S6 GE. The study protocol was approved by the local ethics committee.

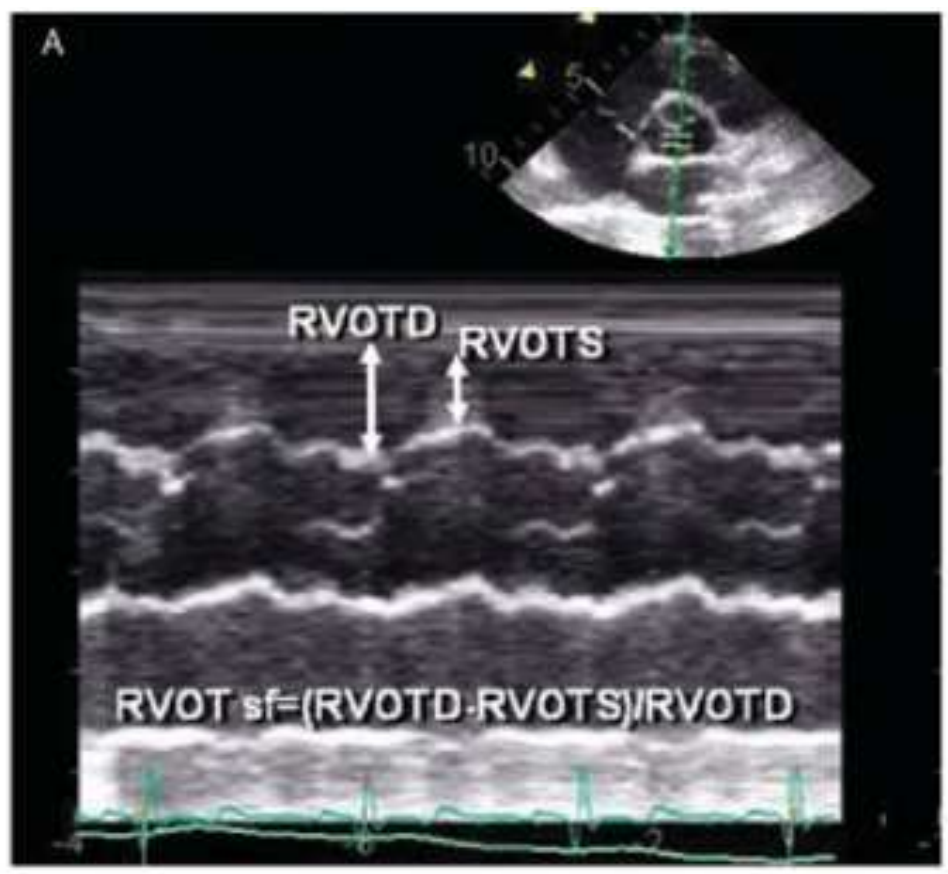

Figure 1.M-Mode 2D Echocardiography from parasternal short-axis view at aortic valve level 
RVOT FS was measured by M-mode from parasternal short-axis view at aortic valve level with magnified images of RVOT, and the cursor was aligned perpendicular to the anterior RVOT wall. Then, RVOT end-diastolic (RVOT ED) and RVOT end-systolic (RVOT ES) diameters were measured. RVOT FS was defined as RVOT FS $=100 \% \times($ RVOT ED - RVOT ES $) /($ RVOT ED $)$.

All data were analyzed using SPSS software, version 19.The categorical variable is presented with the number or frequency (n) and percentage (\%). The numerical variables are assigned with mean (mean) and standard deviation values for normally distributed data. Normality test in numerical variables of all subjects using one sample Kolmogorov Smirnov with $n>50$. Cut off point of numerical data was obtained from ROC. Comparison between dependent variable and independent variable was assessed by Pearson Chi Square. Statistical data analysis using statistical software, $p<0.05$ is considered to be statistically significant.

\section{Results}

The total number of study subjects was 84 people, consisting of 43 people with normal right ventricular function and 41 people with impaired right ventricular function. There were 33 men (80.5\%) and 8 women (29.5\%) with impaired RV function and there was no significant different in both. The mean age of impaired RV function group was 54 years. The mean RVOT FS in normal RV function group was $35.27 \%$ and $20.86 \%$ in impaired RV function group and significantly different between both group $(P<0.001)$.

Table 1. Baseline Characteristics between both study groups

\begin{tabular}{|l|l|l|l|}
\hline \multicolumn{1}{|c|}{ Parameter } & \multicolumn{1}{c|}{$\begin{array}{c}\text { RV FAC } \mathbf{3 5} \text { \% } \\
\mathbf{N} \mathbf{4 3}\end{array}$} & $\begin{array}{l}\text { RV FAC <35\% } \\
\mathbf{N = 4 1}\end{array}$ & $\begin{array}{l}\text { P } \\
\text { Value }\end{array}$ \\
\hline $\begin{array}{l}\text { Male } \\
\text { Female }\end{array}$ & $\begin{array}{l}34(79,1) \\
9(20,9)\end{array}$ & $\begin{array}{l}33(80,5) \\
8(29,5)\end{array}$ & 0.872 \\
\hline Age (years) & $54.98 \pm 10.08$ & $54.37 \pm 9.69$ & 0.778 \\
\hline LVEF (\%) & $37.72 \pm 8.16$ & $25.8 \pm 6.9$ & $<0.001$ \\
\hline LVEDD (mm) & $53.4 \pm 6.15$ & $60.15 \pm 6.25$ & $<0.001$ \\
\hline RA mayor (mm) & $33.09 \pm 5.23$ & $49.41 \pm 7.18$ & $<0.001$ \\
\hline RA minor $(\mathbf{m m})$ & $33.09 \pm 5.22$ & $40.15 \pm 6.30$ & $<0.001$ \\
\hline RV Basal (mm) & $36.53 \pm 5.3$ & $42.66 \pm 5.15$ & $<0.001$ \\
\hline E/A & $1.31 \pm 0.78$ & $2.067 \pm 0.93$ & $<0.001$ \\
\hline RVFAC (\%) & $45.37 \pm 7.25$ & $26.81 \pm 4.69$ & $<0.001$ \\
\hline TAPSE (mm) & $19.95 \pm 2.54$ & $14.4 \pm 2.5$ & $<0.001$ \\
\hline S' (mm/s) & $12.21 \pm 2.08$ & $8.68 \pm 1.72$ & $<0.001$ \\
\hline RVOTFS (\%) & $35.27 \pm 8.22$ & $20.86 \pm 7.33$ & $<0.001$ \\
\hline RVOTSE (mm) & $5.95 \pm 1.39$ & $3.07 \pm 1.43$ & $<0.001$ \\
\hline $\begin{array}{l}\text { Caused : } \\
\text { Coronary heart disease } \\
\text { Hypertension heart disease } \\
\text { Cardiomyopathy }\end{array}$ & $32(74.4 \%)$ & $31(75.6 \%)$ & \\
\hline
\end{tabular}

By using ROC curve, we could find the area under the curve (AUC) from RVOT FS value, which showed RV function in hear failure population. In this study, we found the AUC was 0.919 with P value < 0.001. It shows that RVOT FS was significant as echocardiography parameter to evaluate RV function in heart failure. The cut off point is $\leq 25.5 \%$ shows impaired RV function with $88.4 \%$ sensitivity and $82.9 \%$ specificity. 


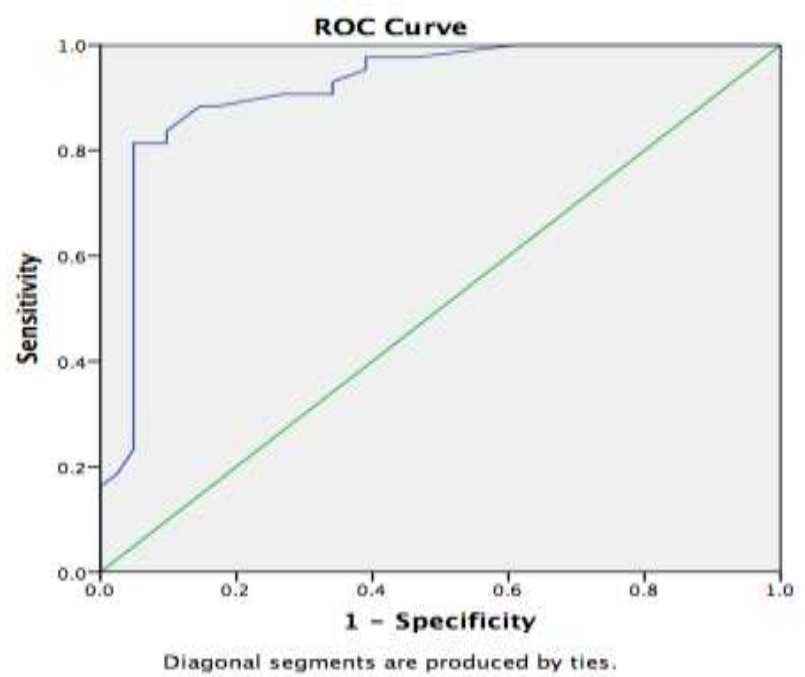

\section{Picture 2. ROC curve RVOT FS}

We correlate RVOT FS, TAPSE and S' with RV FAC value. RVOT FS showed positive correlation of high significance with RV FAC $(\mathrm{r}=0.644, \mathrm{P}<0.001)$. TAPSE and $\mathrm{S}$ ' also showed positive correlation of high significance with RV FAC $(r=0.788, \mathrm{P}<0.001)$ dan $(\mathrm{r}=0.703, \mathrm{P}<0.001)$. (Table 2)

By using ROC curve, RVOT FS, TAPSE and S' showed positive correlation with RV FAC and TAPSE has the best AUC value and the value is 0.963. (figure 3)

Table 2. Correlation RVOT FS, TAPSE and S' with RV FAC value

\begin{tabular}{|l|l|l|}
\hline Parameter & $\begin{array}{l}\text { Coefficient Correlation } \\
(\mathbf{r})\end{array}$ & P value \\
\hline RVOTFS & 0.644 & $<0.001$ \\
\hline TAPSE & 0.788 & $<0.001$ \\
\hline S & 0.703 & $<0.001$ \\
\hline
\end{tabular}

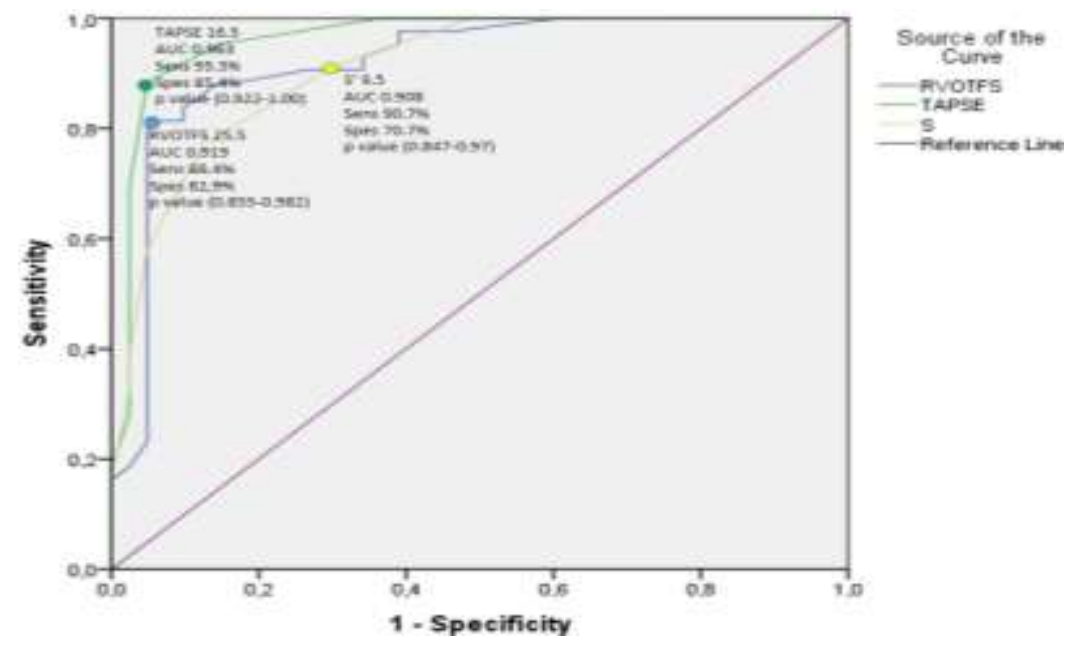

Figure 3. ROC curve RVOT FS, TAPSE, and S' with RV FAC value

\section{Discussion}

Echocardiography assessment of RV function is the important parameter for understanding pathophysiology of heart failure ${ }^{1,3,4}$ However, it is still challenging to find a simple and comprehensive parameter by echocardiography. In baseline characteristic, we found significant difference ejection fraction 
between normal RV function and impaired RV function. The ejection fraction in impaired RV function group was $25.8 \pm 6.9$ and other group was $37.72 \pm 8$.16.The difference dimension of heart chamber in left ventricle, right atrium and right ventricle also hassignificance difference in both of group with $\mathrm{P}$ value $<0.001$.

In the current study by Yamaguchi et al, we found significant difference size of LA, LV, RA and RV in both of group because there was interrelationship between both ventricle. ${ }^{1,10}$ Right heart failure can occurred due to left ventricular dysfunction. Because of increased Left Ventricle End Diastolic Pressure (LVEDP) cause secondary pulmonary hypertension due to increase filling pressure in left ventricle. Unlike left ventricle, RV has anatomical structure which more sensitive with pressure load. ${ }^{2,5,11,12}$

In this study, RVOT FS showed positive correlation with RV FAC in heart failure population. RVOT FS $\leq 25.5 \%$ showed impaired RV function with $88.4 \%$ sensitivity and $82.9 \%$ specificity. The similar results were reported by Lamyaa et al, that RVOT FS $\leq 32 \%$ showed impaired RV with $93 \%$ sensitive and $98 \%$ specific. Original study by Lindqvist et al, showed that RVOT FS with several RV functional parameters, and our data support that RVOT-FS positively correlated with RVFAC. It shows that RVOT FS can diagnose impaired RV function. Because RV has three part of anatomy that contribute in contraction of RV, i.e., the inlet, trabeculate and infundibulum or outflow tract compartment. ${ }^{5}$ RVOT contribute $20 \%$ of RV contraction and $15 \%$ stroke volume of $\mathrm{RV}$, $^{7,11,13}$

In our study, we correlate the other RV function parameter such as RVOT FS, TAPSE and S' with RV FAC. We found that TAPSE had high positive correlation than other $(\mathrm{r}=0.788)(\mathrm{P}<0.001)$. The similar findings were reported by Elmayee, et al that TAPSE has strong correlation with RV FAC $(r=0.72)(\mathrm{P}=0.02)$.

\section{Study Limitation}

This study comes from single medical center with a relative small number of patients. We correlate RVOT FS with RV FAC, we don't correlate it with the gold standart of RV function MRI

\section{Conclusion}

RVOT FS is new parameter echocardiography to evaluate RV function in heart failure population which easy, simple and applicable in clinical practice.

\section{References}

1. Yamaguchi M, Toshihiro T, Yuki W, dkk. Reduced fractional shortening of right ventricular outflow tract is associated with adverse outcomes in patients with left ventricular dysfunction, Cardiovascular Ultrasound, 2013, 11:19.doi:10.1186/1476-7120-11-19.

2. Clifford R, Greyson MD. Pathophysiology of Right Ventricular Failure, Critical Care Medicine, 2008; 36(1)

3. Antoine Kossaify. Echocardiographic Assessment of the Right Ventricle, from the Conventional Approach to Speckle Tracking and Three-Dimensional Imaging, and Insights into the "Right Way" to Explore the Forgotten Chamber. Echocardiography unit, cardiology division, University Hospital Notre Dame de Secours, Byblos, Lebanon. 2015.

4. Asmer I, Salim A, Majdi et al. Right ventricular outflow tract systolic excursion: a novel echocardiographic parameter of right ventricular function, European Heart Journal, 2012; 13: 871877.

5. Bulent D, Kazim B, Murat G, et al. Right Ventricular Outflow Tract Function In Chronic Heart Failure, Indian Heart Journal, 2016;68(suppl 1); S10-S14. doi:10.1016/j.ihj.2015.07.028.

6. Dell'Italia LJ, Walsh RA. Anatomy and Physiology of the Right Ventricle, American Heart Journal, 1998; 116:1289-97. doi:10.1016/0002-8703(88)90454-1.

7. Hayrapetyan, Hamlet. Anatomical and Physiological Patterns of Right Ventricle, Journal of Cardiology and Current Research, 2015; 2(1)

8. Haddad F, Sharon A.H, David NR, dkk.Right Ventricular Function in Cardiovascular Disease, Part I Anatomy, Physiology, Aging, and Functional Assessment of the Right Ventricle, Guidelines for the Echocardiographic Assessment of the Right Heart in Adults,2010; 23:685-713.

9. Jurcut R, Sorin G, Andre L G, Simon V, The Echocardiographic Assessment Of The Right 
Ventricle: What To Do In 2010,European Journal of Echocardiography, 2010; 11, 81-96

10. Lawrence G, Rudski, Wayman C W, Jonathan A, dkk. Guidelines of Echocardiography Assessment of the Right Heart in Adults: A Reports From the American Society of Echocardiography, Journal of American Society of Echocardiography,2010; 23:685-713. doi:10.1016/j.echo.2010.05.010.

11. Lindqist V, M Heinen, Kazzam E. Right Ventricular Otflow Tract Fractional Shortening An Applicable Measure In Right Ventricular Function,European Journal of Echocardiography, 2003; 4:29-35. doi:10.1053/euje.4.1.29.

12. Michael P D, Shivani M B, Laura M R. How Best to Assess Right Ventricular Function by Echocardiography,Cardiology in the Young, 2015 December; 25(8): 1473-1481. doi:10.1017/S1047951115002255.

13. Tanya D, Wilbert S Aronow. Echocardiographic Evaluation of The Right Ventricle: Clinical Implication, Clinical Cardiology, 2017; 40:542-548. doi:10.1002/clc.22694.

$* * * * * * * * * *$ 